\title{
Sizes and Structures of Micelles of Cationic Octadecyl Trimethyl Ammonium Chloride and Anionic Ammonium Dodecyl Sulfate Surfactants in Aqueous Solutions
}

\author{
Hong-Un Kim and Kỵung-Hee Lim* \\ Department of Chemical Engineering, Chung-Ang Chiversin, Seoul 156-756, Korea \\ Received Mav 9, 2003
}

\begin{abstract}
The sizes and stnictures of micelles formed in aqueous solutions of cationic octadecyl trimethyl ammonium chloride (OTAC) and anionic ammonium dodecyl sulfate (ADS) surfactants were investigated using smallangle neutron scattering (SANS), self-diffusion coefficients by pulsed-gradient spin-echo (PGSE) NMR, and dynamic light scattering (DLS) methods. SANS and DLS data indicate that their stnuctures are spherical at concentrations as high as $300 \mathrm{mM}$. As the total surfactant concentration increases. the peaks of SANS spectra shift to higher scattering vector and become sharper. indicating that the intermicellar distance decreases and its distribution becomes narrower. This is due to more compact packing of surfactant molecules at high concentrations. The intermicellar distance of around $100 \AA$ above $200 \mathrm{mM}$ corresponds approvimately to the diameter of one micelle. The sizes of spherical micelles are $61 \AA$ and $41 \AA$ for $9 \mathrm{mM}$ OTAC and $10 \mathrm{mM}$ ADS. respectively. Also the self-diffision coefficients by PGSE-NMR yield the apparent sizes $96 \AA$ and $31 \AA$ for micelles of $1 \mathrm{mM}$ OTAC and $10 \mathrm{mM}$ ADS. respectively. For ADS solutions of high concentrations (100-300 $\mathrm{mM}$ ). DLS data show that the micelle size remains constant at $25 \pm 2 \AA$. This indicates that the transition in micellar shape does not take place up to $300 \mathrm{mM}$. which is consistent with the SANS results.
\end{abstract}

Key Words : Ammonium dodecyl sulfate (ADS). Octadecyl trimethyl ammonium chloride (OTAC), Smallangle neutron scattering (SANS), Pulsed-gradient spin-echo (PGSE)-NMR. Intermicellar distance

\section{Introduction}

One of the characteristics of surfactants is the capacity to form association structures in solutions. Micelles are one type of such structures. and the minimum concentration to form micelles is called the critical micelle concentration (CMC). The CMC is not a specific concentration but a narrow range of concentrations. The micellar solutions above CMC are homogeneous and transparent, thus it is difficult to observe size and structure of micelles. In general. it is known that the size of micelles formed in aqueous surfactant solution ranges from 10 to $100 \AA$. and that the shape of micelles varies with increasing concentration in order of spherical. cylindrical, hexagonal, and lamellar in aqueous ionic surfactant solutions. . $^{\text {-4 }}$

Snall-angle neutron scattering (SANS) can probe interfacial structures of surfactant aggregations, polymers. and biomolecules, utilizing the neutron scattering intensity difference between lydrogen and deuterium. A good permeability and no charge of neutrons favor internal structure analysis. Therefore, SANS has been widely used for the determination of sizes and structures of micelles.

Pulsed-gradient spin-echo (PGSE) NMR spectrometry has the ability to measure the self-diffusion coefficients of monomers and micelles in aqueous surfactant solutions. which thus enables to probe the interactions between them.

\footnotetext{
"Corresponding Author. Tel: +82-2-820-5275, Fax: +82-2-8263574; e-mail: khlimiấcaulac. kr

†Present address: Department of Applied Chemistry, Dongyang Technical College, Seoul 152-714, Korea
}

The formation of micelles and its phase transition in aqueous surfactant solutions is related to the diffusion of hydrophobic parts in the micellar phase. Thus, the self-diffusion coefficient is a measure of analyzing the molecular organization in solutions and is highly affected by the changes in structure and association/dissociation in colloidal dispersions. ${ }^{6}$ The self-diffusion coefficient $\left(D_{s}\right)$ in aqueous surfactant solutions decreases with increasing surfactant concentration, which is due to the hindrance of diffusion by interaction between surfactant monomers. Also self-diffusion of micelles proceeds slowly since the size of micelles is larger than that of monomers. As the surfactant concentration increases. the transition of shape from spherical to cylindrical contributes to the reduction of self-diffusion coefficient, too.

We reported previously the $\mathrm{CMC}$, its dependence on temperature, and thermodynamics of micellization for the cationic octadecyl trimethyl anmonium chloride (OTAC) and anionic ammonium dodecyl sulfate (ADS) ${ }^{7}$ OTAC has been widely used in industries due to excellent antistatic effects and softness, and ADS has been used in cosmetics and personal cares in place of the well-known sodium dodecyl sulfate (SDS) which undergoes autocatalytic acid hydrolysis in acidic solutions or at high temperatures and causes skin irritation. No information on sizes and structures of micelles at different concentrations has been obtained for these surfactant systems.

The sizes. shapes, and transition of OTAC and ADS micelles in aqueous solutions have been investigated using SANS. self-diffision coefficient by PGSE-NMR and dynamic light scattering (DLS). and their results are reported in this article. 


\section{Experimental Section}

Materials. The anionic ammonium dodecyl sulfate (ADS) and cationic octadecyl trinethyl anumonium chloride (OTAC) surfactants were purchased from Fluka. ADS was in the form of a $30 \%$ aqueous solution. It was placed in a rotary evaporator to reduce the contents of water and volatile materials. Then it was recrystallized with $90 \%$ ethanol and absolute ethanol twice, respectively. The OTAC had a stated purity of $98 \%$ and was recrystallized three times with absolute ethanol. The purified surfactants were finally dried in an evacuated desiccator. ${ }^{8}$ All samples for SANS and PGSE-NMR measurements were prepared in $\mathrm{D}_{2} \mathrm{O}$ (Aldrich. purity 99.9 atom $\% \mathrm{D}$ ).

Small-angle neutron scattering (SANS). The fundamental principles of SANS are that the scattering vector. $q$. corresponds to the change in neutron momentum and that the scattered intensity. $I_{s}(q)$. changes with the sample. The scattering vector is defined as

$$
q=|\vec{q}|=\frac{4 \pi}{\lambda} \sin \left(\frac{\theta}{2}\right)
$$

where $\lambda$ and $\theta$ are the wavelength and scattered angle of neutron. The intensity of neutron scattered is given by

$$
I_{s}(q)=\Phi A d T_{S+S B}\left(\frac{d \Sigma}{d \Omega}(\vec{q})\right)_{S} \Delta \Omega \varepsilon t
$$

Here, $\Phi$ is the neutron flux unto the sample, $A$ sample area. $d$ sample thickness. $T_{S-S B}$ transmission through the sample plus sample cell, $\Sigma$ the scattering cross area. $\Omega$ the solid angle subtended by one pixel of the detector, $\varepsilon$ detector efficiency. and $t$ effective counting time, respectively. The absolute scattering cross area $[d \Sigma(\vec{q}) / d \Omega]_{s}$ includes the form factor $P(\vec{q})$ and structure factor $S(\vec{q})$ as well as number density $\left(N_{P}\right)$ of particle as follows.

$$
\left(\frac{d \Sigma}{d \Omega}(\vec{q})\right)_{S}=N_{F} P(\vec{q}) S(\vec{q})
$$

The scattering vector $q$ and the neutron wavelength ranged from 0.006 to $0.6 \AA^{-1}$ and 4 to $8 \AA$. respectively. The resolution of wavelengths was $10 \%$ with full width at halfmaximum value. The samples prepared in $D_{2} O$ were injected carefully without making bubbles into quartz cylindrical cells (Next Instrument $\mathrm{Co}_{0}$.) with a path length of $2 \mathrm{~mm}$ and outside diameter of $22 \mathrm{~mm}$. The calibration of absolute scattering intensity was carried out using silica and the scattering intensity was normalized by dividing by that of $D_{\searrow} O$. The measured data were treated according to the procedures of Hantaro of Korea Atomic Energy Research Institute (KAERI). ${ }^{9}$

The scattering intensity $I_{s}(q)$ measured of the sample and the Guinier law, equation (4). yield the radius of gyration $\left(R_{g}\right) .^{1(i)}$

$$
I_{s}(q)=I(0) \exp \left(\frac{-R_{g}^{2}}{3} q^{2}\right) \quad \text { for } 0.5<q R_{\varepsilon}<1.4
$$

where $I_{s}(q)$ and $I(0)$ are the intensities of scattered neutrons with and without sample. respectively. which are functions of scattering vector $q$. The radius $\left(R_{m c}\right)$ of spherical micelles is calculated from the relation. ${ }^{11}$

$$
R_{m i c}=\sqrt{5 / 3} R_{g}
$$

The shape of micelles can be determined using $n$ values of the power-law. $I_{s}(q) \propto q^{-n}$. The spherical micelle is identified when $n=4$ according to Porod s law ${ }^{12}$ Also $n=1$ points to the cylindrical or rod-like micelles at $1 / l_{p}<q<$ $1 / R_{\text {mic }}$ where $i_{p}$ is the length of cylindrical micelles. ${ }^{12.14}$

The length $l_{p}$ can be determined from the Holtzer plot or bending rod plot in which $q I_{5}(q)$ is plotted versus $q^{13,15.16}$ That is, $l_{P}$ is obtained from $q l_{P} \approx 1.9$ for which the $q$ value is determined from the intersection point of linear fits for low and high $q$ values.

Measurements of self-diffusion coefficients by PGSENMR. The self-diffusion coefficients $\left(D_{s}\right)$ of monomer and micelle were measured using the pulsed-gradient spin-echo (PGSE) NMR with a Bruker DMX 600 spectrometer. The echo attenuation intensity $A(g)$ as a function of gradient amplitude $(g)$ is expressed by equation (6), as was found to decay exponentially.

$$
A(g)=A(0) \exp \left[-\gamma^{2} \delta(\Delta-\delta / 3) D_{s} g^{2}\right]
$$

Here. $A(0)$ is the echo intensity in the absence of gradient. $\gamma$ is the gyromagnetic ratio of proton. whose value is given by $2.675 \times 10^{8} \mathrm{~T}^{-1} \mathrm{~s}^{-1}{ }^{17}$ Also $\delta$ and $\Delta$ are pulsed gradient duration and the time interval between two gradient pulses, respectively. as depicted in Figure $1^{18-20}$ In Figure 1 , it is shown that two radio frequency pulses and two field gradient pulses of magnitude $g$. duration $\delta$, separation $\Delta$ were applied before and after the $180^{\circ}$ refocusing pulse. The second half of the spin echo was Fourier-transformed. and the relative intensities of the resolved absorption peaks were measured.

Values of $D_{s}$ were determined from the slope of $\ln (A(g) /$ $A(0))$ versus $g^{2}$ plots at fixed $\delta$ and $\Delta$ with varying $g$ in equation (6). ${ }^{2.22}$ Calibration was carried out with $D_{2} O$ and its self-diffusivity was measured to be $1.865 \times 10^{-9} \mathrm{~m}^{2} / \mathrm{s}$. which is in excellent agreement with the literature value. $1.872 \times 10^{-9} \mathrm{~m}^{2} / \mathrm{s}^{2.3}$ Based on this calibration result. $D_{s}$ of methanol at $25^{\circ} \mathrm{C}$ was determined as $1.98 \times 10^{-9} \mathrm{~m}^{2} / \mathrm{s}$ from the slope -31.164 for $\delta=4 \mathrm{~ms}$ and $\Delta=15.1 \mathrm{~ms}$. This value is

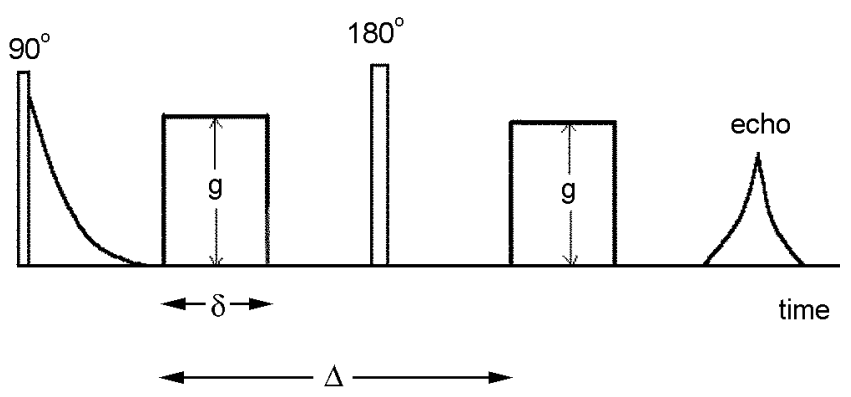

Figure 1. Schematic of the pulsed-gradient spin-echo pulse sequence. 


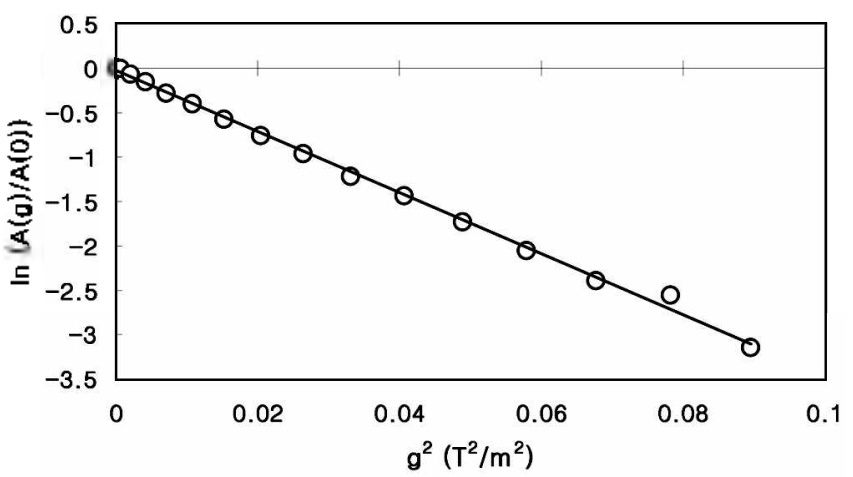

Figure 2. Plot of $\ln (A(g) / A(0))$ versus $g^{2}$ tor the determination of $D_{s}$ at $25^{\circ} \mathrm{C}$ for $1 \mathrm{mM}$ ADS solution. The diffusivity $D_{s}$ is calculated from the slope $(=-34.367)$ : the conelation coefticient is 0.9987 .

compared well with that of literature. $2.30 \times 10^{-19} \mathrm{~m}^{2} / \mathrm{s}$. $2 . .5$

Figure 2 shows an example of how to determine $D_{s}$ from equation (6) for the $1 \mathrm{mM} \mathrm{ADS}$ solution. The self-diffusion coefficients of monomers. $D_{s}^{\text {mors }}$. and of nicelles, $D_{s}{ }^{1 /}$, were measured directly at concentrations below and above the CMC by PGSE-NMR. The NMR peaks characteristic to the surfactant was observed conspicuously at these concentrations

Dynamic light scattering (DLS). In order to determine the sizes and their distribution of OTAC and ADS micelles. scattered dynamic light intensities were measured using a ZetaSizer 1000HS (Malven Instruments Ltd. UK) with a capillary cell. Brownian motions of the micelles were observed by DLS and related to their sizes.

The detector angle of 90 degrees, temperature of $25^{\circ} \mathrm{C}$. and wavelength of $633 \mathrm{~mm}$ were set. The refractive indices of OTAC (1.44), $\operatorname{ADS}(1.37)$, and solvent deionized (D.I.) water (1.33). and the viscosity of solvent $(0.891 \mathrm{cP})$ were used. All samples were prepared in D.I. and triply distilled water.

\section{Results and Discussion}

Size and structure of micelles by SANS. The SANS spectra of $9 \mathrm{mM}$ OTAC and $10 \mathrm{mM}$ ADS solutions in $D_{2} O$ were measured and the plots of $\log (q)-\log \left(I_{s}(q)\right)$ are made. One of such plots is shown in Figure 3. The slopes at high $q$ s are -3.994 and -3.902 . respectively. These values are very close to -4 , which signifies that the Porod's law, $I_{s}(q) \propto q^{-4}$ is satisfied. These results point to the fact that the micelles in the aqueous $9 \mathrm{~mm}$ OTAC and $10 \mathrm{mM}$ ADS solutions are spherical.

Surfactant association structures may be explained by a geometric analysis, i.e., by the values of critical packing parameter. $N_{F}=V / a_{0} l={ }^{6}$ Here $V$ and $/$ are the volume and length of the alkyl chain of the surfactant and they are usually calculated from $V=\left(27.4+26.9 N_{c}\right) \AA^{3}$ and $I=$ $\left(1.54+1.265 N_{\mathrm{L}}\right) \AA \times 0.77$ with $N_{\mathrm{L}}$ being the carbon number in the hydrophobic chain of the surfactant. ${ }^{27}$ The factor 0.77 in the length / reflects that the real length is essentially $77 \%$ of fully extended length of the carbon chain. The quantity $\alpha_{\mathrm{c}}$
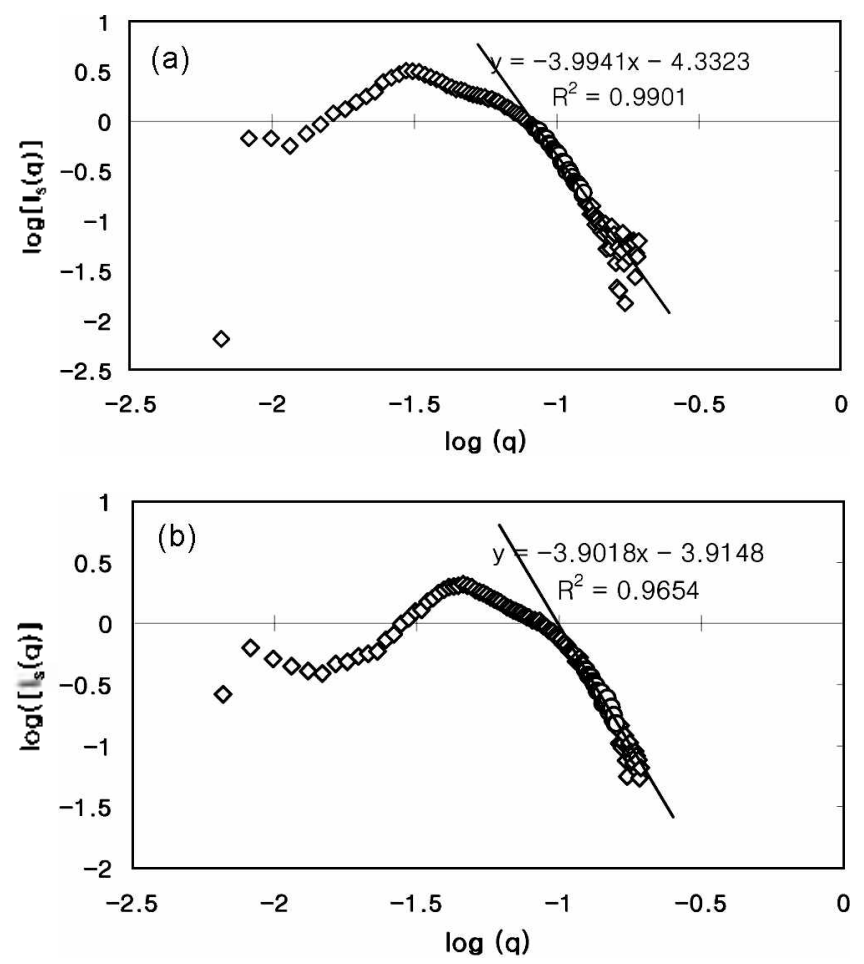

Figure 3. Plot of $\log (q)$ versus $\log \left(I_{s}(q)\right)$ and their linear fits at high $q$ for $9 \mathrm{mMOTAC}(\mathrm{a})$ and $10 \mathrm{mM} \mathrm{ADS}(b)$.

is the area of the surfactant head group and is determined from the slope of surface tension versus concentration, $\gamma-$ $\log C$. plots. For OTAC and $\mathrm{ADS}, N_{P}$ values are 0.2 and 0.37 calculated from the $\gamma-\log C$ data ${ }^{2}$ These values suggest that the OTAC and ADS molecules be conical in shape and that since $N_{p},<1 / 3$ or $N_{p}, \sim 1 / 3$, their micelles be spherical in aqueous solutions, ${ }^{29}$ which is in good agreement with SANS data.

In order to determine the sizes of micelles. plots of $q^{2}$ versus $\ln \left(I_{s}(q)\right)$ were made and one example of such plots is shown in Figure 4. The radii of gyration for $9 \mathrm{mM} \mathrm{OTAC}$ and $10 \mathrm{mM}$ ADS are obtained as $23.6 \AA\left(0.6<q R_{g}<1.4\right)$ and $16 \AA\left(0.5<q R_{g}<1.3\right){ }^{10}$ respectively, from the slopes of linear fits (Figure 4 ). The radii of spherical micelles $\left(R_{m i c}\right)$ are then determined as 30.5 and $20.7 \AA$ for $9 \mathrm{mM} \mathrm{OTAC}$ and $10 \mathrm{mM}$ ADS solutions. respectively, from $R_{m i c}=\sqrt{5 / 3} R_{\mathrm{g}}$. The radius ( $20.7 \AA$ ) of ADS spherical micelles is larger than that ( $18 \AA$ ) of the sodium dodecyl sulfate (SDS) measured by SANS at dilute aqueous solutions. ${ }^{3(1.3]}$ In ADS and SDS nicelles, the counterion $\left(\mathrm{NH}_{4}^{-}\right)$in the fomer is larger than that $\left(\mathrm{Na}^{-}\right)$in the latter. This is why the size of the ADS micelles is larger.

The sizes and structural transitions of ADS and OTAC micelles were examined by SANS at various surfactant concentrations and the SANS results are shown in Figure 5. Both surfactant systems exhibit peaks in intensity. which means. according to Grabner et al..$^{32}$ that spherical micelles were formed at the concentrations examined. If the shape of the micelles were cylindrical. plateaus (instead of peaks) would appear in the SANS spectra. ${ }^{32.33}$ Hence the results of 

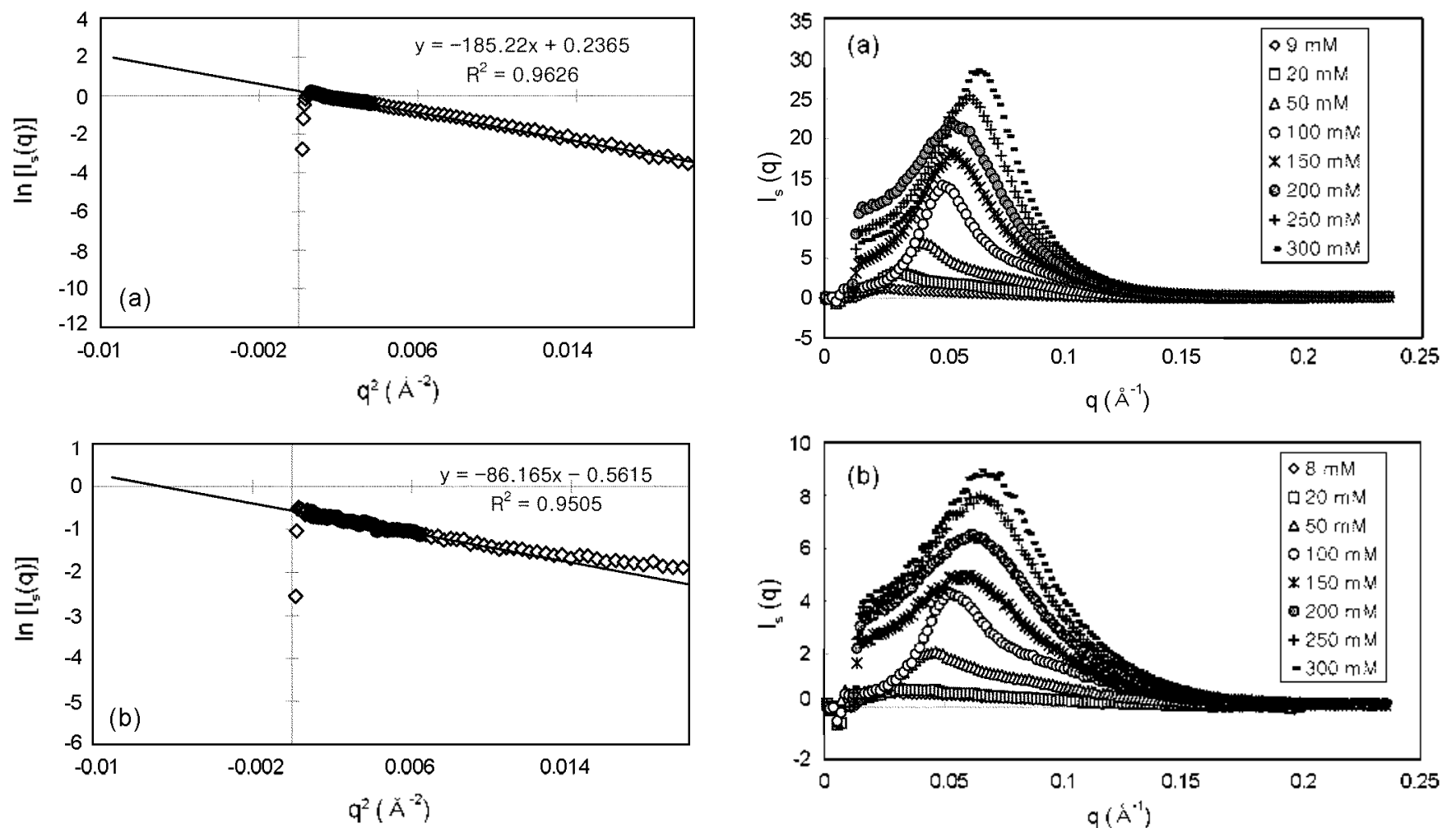

Figure 4 . Guinier plot of $q^{2}-\ln (s+q)$ and determination of gyration radius for $9 \mathrm{mMOTAC}(\mathrm{a})$ and $10 \mathrm{mM} \mathrm{ADS}(\mathrm{b})$.

Figure 5 imply that no structural transition from spherical to cylindrical micelles was observed up to $300 \mathrm{mM}$. In contrast. such transition was observed at $250 \mathrm{mIM}$ for SDS micelles. exanined by X-ray scattering. ${ }^{34}$

The locations of the peaks correspond to the intermicellar distance. The position of peaks shifts toward high $q$, as surfactant concentration increases. This result indicates that the intermicellar distance (calculated from $2 \pi / q_{p e a k}$ ) becomes smaller. as repulsive interactions between micelles become stronger at higher concentrations. On the other hand. the micelle sizes change little with surfactant concentrations up to $50 \mathrm{mM}$. The micelle sizes and intermicellar distances at various surfactant concentrations are presented in Table 1.

With increasing surfactant concentrations. the intermicellar

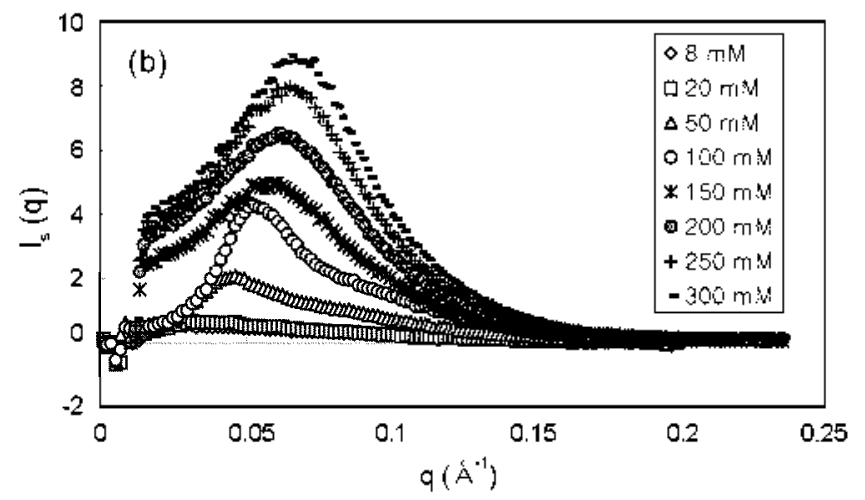

Figure 5. SANS spectra of aqueous solutions of OTAC (a) and $\mathrm{ADS}(\mathrm{b})$ at various surfactant concentrations.

distance decreases significantly up to $50 \mathrm{mM}$ and then the rate of decrease is reduced substantially. Considering the diameters of a micelle ( 61 and $41 \AA$ for OTAC and ADS micelles. respectively). the intermicellar distance at high surfactant concentrations correspond to the sizes of one or two nucelles. Structural transition from spherical to cylindrical micelles may occur, when the head groups of neighboring micelles interact repulsively each other (Figure 6). However, for OTAC and ADS micelles. the intermicellar distances are large enough for micellar interactions to be weak and therefore for the structural transition not to occur.

The peaks become sharper with increasing surfactant concentration. This means that the distribution of intermicellar distance is narrower due to stronger repulsive

Table 1. Micelle sizes and intermicellar distances at various concentrations of cationic OTAC and anionic ADS surfactants

\begin{tabular}{|c|c|c|c|c|c|}
\hline \multicolumn{3}{|c|}{ OTAC } & \multicolumn{3}{|c|}{$\mathrm{ADS}$} \\
\hline $\begin{array}{l}\text { Concentration } \\
\text { (mM) }\end{array}$ & $\begin{array}{c}\text { Diameter } \\
(\AA)\end{array}$ & $\begin{array}{c}\text { Internicallar distance } \\
(\bar{A})\end{array}$ & $\begin{array}{c}\text { Concentration } \\
(\mathrm{mM})\end{array}$ & Diameter $(\AA)$ & $\begin{array}{c}\text { Intermicellar distance } \\
(A)\end{array}$ \\
\hline 9 & $61 \pm 4$ & 246 & 10 & $41 \pm 4$ & 347 \\
\hline 20 & $60 \pm 4$ & 213 & 20 & $44 \pm 4$ & 201 \\
\hline 50 & $60 \pm 6$ & 153 & 50 & $44 \pm 4$ & 136 \\
\hline 100 & - & 127 & 100 & - & 119 \\
\hline 150 & - & 118 & 150 & - & 112 \\
\hline 200 & - & 121 & 200 & - & 101 \\
\hline 250 & - & 104 & 250 & - & 97 \\
\hline 300 & - & 99 & 300 & - & 97 \\
\hline
\end{tabular}




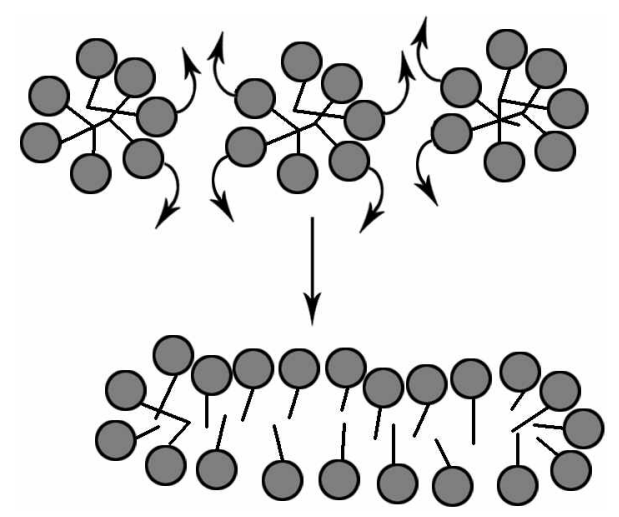

Figure 6. Schematic of structural transition from spherical to cylindrical micelles.

interaction antong micelles when the solution becones more crowded with micelles. ${ }^{33}$

NMR spectra and determination of self-diffusion coefficients. Figure 7 shows the NMR spectra of $1 \mathrm{mM}$ OTAC $(C M C=0.33 \mathrm{mM})$ and $10 \mathrm{mM}$ ADS $(C M C=6.5$ $\mathrm{mM}$ ) solutions above $\mathrm{CMCs}$ along with their respective structural formula. In Figure 7a the NMR peaks of OTAC were observed at $0.836\left(\mathrm{CH}_{3}\right.$ functional group $), 1.213$ $\left(\left(\mathrm{CH}_{2}\right)_{1 \leq}\right.$ alkyl chain group). $1.69\left(\mathrm{CH}_{3}(\mathrm{~b})\right), 3.006\left(\mathrm{~N}\left(\mathrm{CH}_{3}\right)_{3}\right)$. $3.23 \mathrm{ppm}\left(\mathrm{CH}_{\smile}(\mathrm{a})\right)$, respectively. Similarly for $\mathrm{ADS}$. the peaks were observed at $0.836\left(\mathrm{CH}_{3}\right.$ functional group $), 1.213$ $\left(\left(\mathrm{CH}_{2}\right)_{1 \leq}\right.$ alkỵl chain group), $1.69\left(\mathrm{CH}_{\star}(\mathrm{b})\right), 3.995 \mathrm{ppm}$ $\left(\left(\mathrm{CH}_{2}(\mathrm{a})\right)\right.$, respectively. and a small peak at $3.62 \mathrm{ppm}$ which corresponds to some impurities. At $0.2 \mathrm{mM}$ OTAC and 1 mM ADS below CMC. NMR peak exhibits the same points as those of micelles. Therefore, we measured the selfdiffusion coefficient directly at the largest of these peaks except for that of alkyl chain group.

Based on the NMR data. the self-diffusion coefficients $D_{s}$ 's of OTAC and ADS were determined at $3.006 \mathrm{ppm}$ $\left(\mathrm{N}\left(\mathrm{CH}_{3}\right)_{3}\right)$ and $0.836 \mathrm{ppm}\left(\mathrm{CH}_{3}\right) . D_{s}$ s were calculated from the slopes of the plots of $\ln (A(g) / A(0))$ versus $g$. Figure 8 shows such plots for OTAC (1 mM) and ADS $(10 \mathrm{mM})$ micelles. The self-diffusivity $D_{s}$ 's calculated from the slopes of the plots are presented in Table 2 .

For $\mathrm{ADS}$, the monomer self-diffusivity $\left(D_{s}^{\text {mon }}\right.$ ) was three times larger than the micelle diffusivity $\left(L_{s}^{\text {in }}\right)$, which reflects the fact that micelles are larger than monomers. For OTAC micelles the diffusivity $D_{s}{ }^{1 / 1}$ is order-of-magnitude smaller than that of $\mathrm{ADS}$ nicelles. This result indicates that the OTAC micelles are larger than the ADS nicelles.

Notably, the OTAC monomer diffusivity measured at 0.2 $\mathrm{mM}$ is approxinately the same as the OTAC micelle diffusivity measured at $1 \mathrm{mM}$. This indicates that $D_{s}^{\operatorname{mon}}$ at 0.2 $\mathrm{mM}$ reflects the diffusivities of many monomers and a few micelles. Previously we reported that CMC of OTAC at 25 ${ }^{6} \mathrm{C}$ was $0.32+\mathrm{mM}$ from the conductivity data. ${ }^{7}$ However. CMC is a narrow concentration range and depends on measurement method. The conductivity method may yield higher $\mathrm{CMC}$. because micelles appeared to be formed at 0.2 mM OTAC according to the NMR data. To avoid such (a)<smiles>CCCCCCCCCCCCCCCCCC[N+](C)(C)C</smiles>

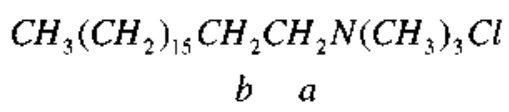

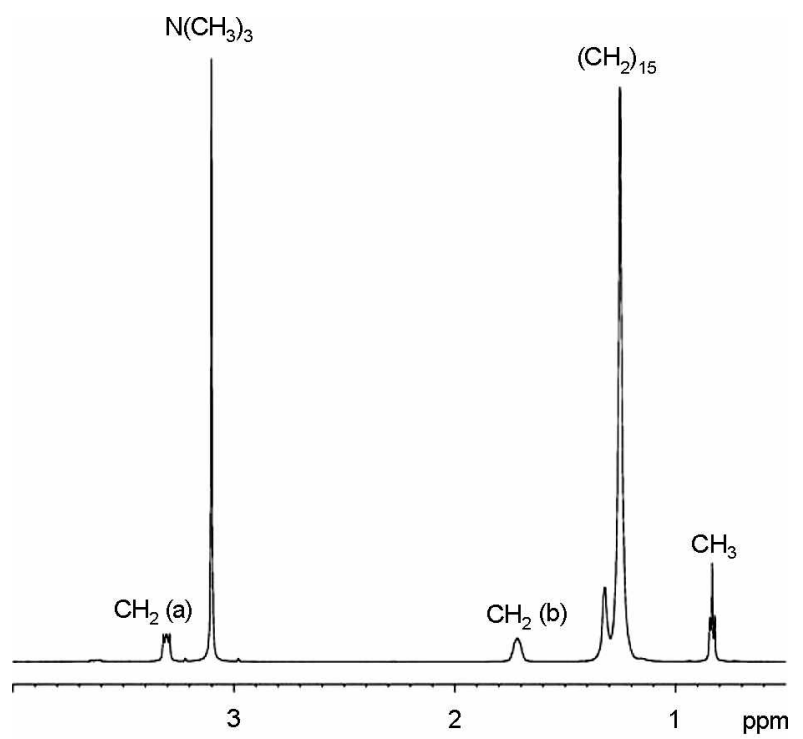

(b)<smiles>[CH-]OS(=O)(=O)OCCCCCCCCCCCC</smiles>

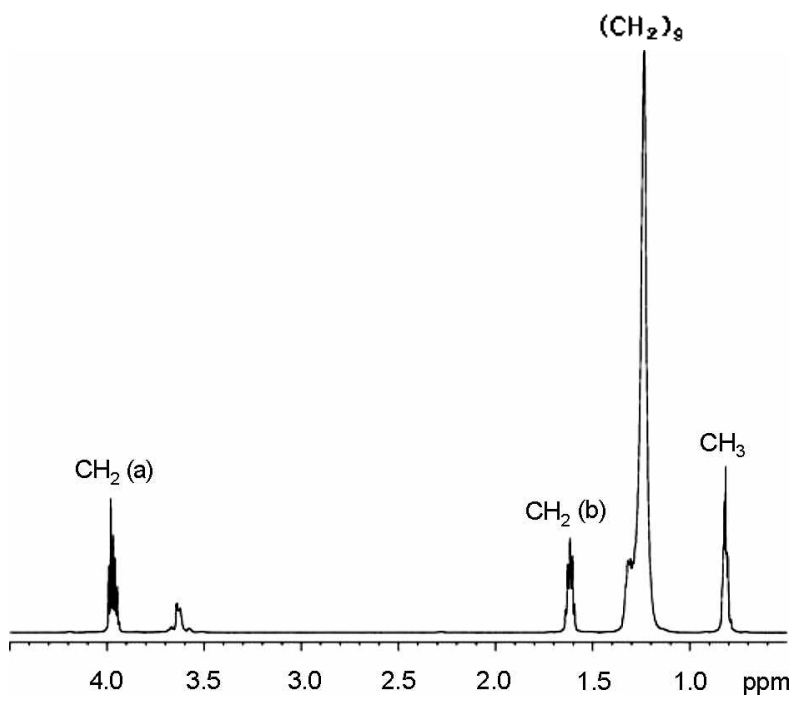

Figure 7. Structural formula and NMR spectra for the aqueous solutions of (a) I mM OTAC and (b) $10 \mathrm{mM}$ ADS

discrepancy. NMR spectra should be obtained at lower surfactant concentrations. That is, when CMC is low. i.e., below $1 \mathrm{mM}$. $D_{s}^{m m}$ is usually determined from $D_{s}{ }^{o b s}$ and equation $(7)$ 


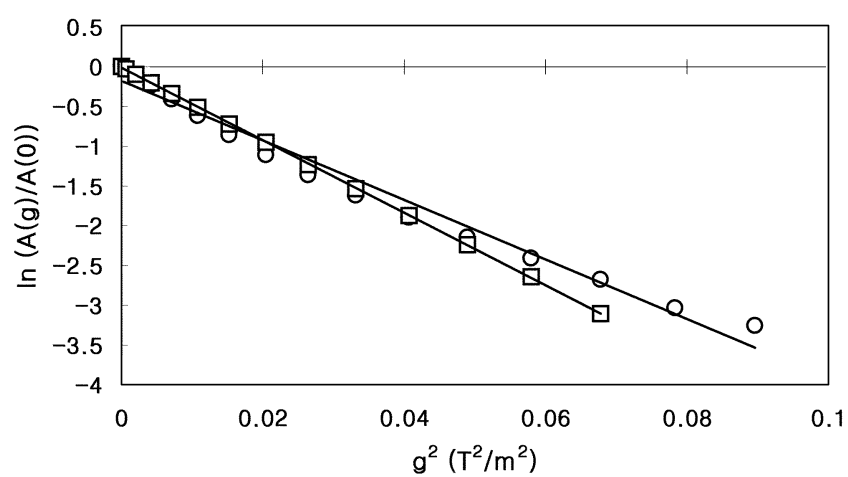

Figure 8. Plot of $\ln (f(g) / f(0))$ versus $g^{2}$ for 1 mM OTAC $($. $)$ and $10 \mathrm{mM}$ AISS aqueous solutions $(0)$ at $25^{\circ} \mathrm{C}$.

Table 2. Sell-dillusion coelicients of monomers $\left(D_{s}^{m+n}\right)$ and micelles $\left.(D)^{M}\right)$ of $O I A C$ and $A D S$ in $D_{2} O$ solutions

\begin{tabular}{ccc}
\hline Self-diffusion cocfficients & OTAC & ADS \\
\hline$D_{0}^{1+40+1}\left(110^{-11} \mathrm{~m}^{2} / \mathrm{s}\right)$ & 6.63 & 42.37 \\
$D_{*}^{t t}\left(\times 10^{-11} \mathrm{~m}^{2} / \mathrm{s}\right)$ & 4.24 & 15.08 \\
\hline
\end{tabular}

$$
D_{s}^{n h s}-D_{s}^{H t}-\frac{C M C}{C_{t}}\left(D_{s}^{m(n)}-D_{x}^{M H}\right)
$$

where $C_{i}$ is the total surfactant concentration, ${ }^{5}$ However, for OTAC this method could not be applied because the NMR signal was too weak for dilute solutions at the prescribed chemical shifts.

As shown in Table 2, the self-diffusion coefficients of micelles are smaller than those of monomers as expected since the size of micelles is larger than that of monomers for both ionic surfactant solutions. The sizes of ADS monomer and micelles are smaller than those of OTAC. thus the selfdiffusion coefficients for ADS monomer and micelles are larger than those for OTAC. Therefore, the values of selfdiffusion coefficients for micelles provide the information on micelle sizes.

The apparent radius of micelles $\left(R_{i \eta p}\right)$ can be estimated from the Stoke-Ëinstein relation which may be expressed as

$$
D_{s}-\frac{k T}{6 \pi \eta R_{a p p}}
$$

where $k$ is the Boltzmann constant. $T$ is the measurement temperature $(298 \mathrm{~K})$, and $\eta$ is the viscosity of the medium, which is considered as almost the same as that of water (1.0 cP).

$R_{\text {imp }}$ of the OJAC micelle at $1 \mathrm{mM}$ solution is $48 \AA$, which is a little larger than that by SANS $(30.5 \dot{A}$ for 9 mM OlAC solution). $R_{\text {rip }}$ of the ADS micelles is $16 \AA$ at $10 \mathrm{mM}$ and is rather smaller than that by SANS $(2 \mathrm{I} \AA)$ at the same concentration. Hence, the self-diffusivity by PGSE-NMR yields sizes different from those by SANS and their difference may be significant $(20-40 \%)$.

Micelle sizes by DLS. Figure 9 shows the size distribution of $\mathrm{ADS}$ micelles in aqueous solutions of high surfactant

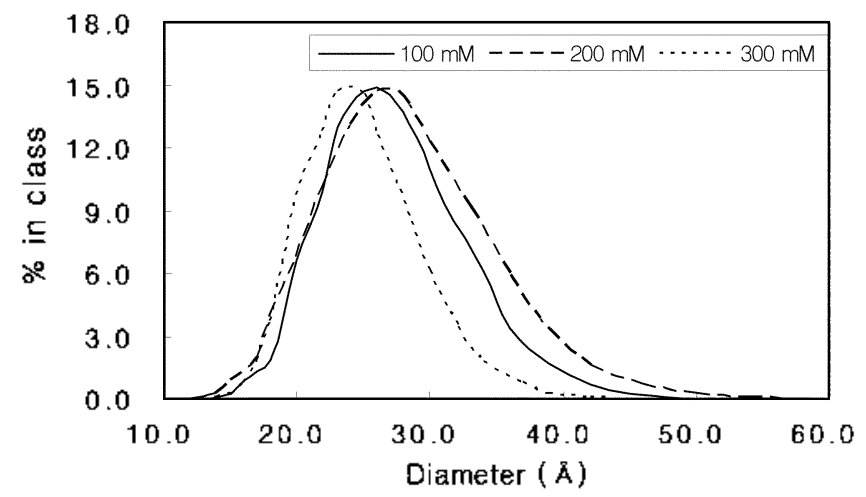

Figure 9. Sire distributions of AISS micelles determined by IDI S at high surfactant concentrations.

concentrations, 100,200, and $300 \mathrm{mM}$. Micelles formed at these high concentrations were monodispersed since their polydispersity ranged firom 0.03 to 0.05 . The average diameter of ADS micelles was estimated as $25=2 \AA$ at these concentrations, which is approximately twice the 77\% (12 $\dot{A})$ of the alkyl chain length of ADS. DIS yielded the smaller micelle size than SANS and PGSE-NMR. At high surfactant concentrations, smaller micelles were obtained probably because of increased interactions between micelles. DIS data also show that the shape transition of ADS micelles did not occur at concentrations up to $300 \mathrm{mM}$, which is in agreement with the SANS result.

Micelle sizes are determined by SANS for dilute solutions of the surfactant concentrations slightly above CMC to minimize interactions between micelles. However, DI SS data are obtained often for concentrated surfactant solutions. For dilute solutions, DISS signals are often too weak to be analyzed for size determination.

\section{Conclusions}

The sizes of micelles of cationic surfactant $O$ TAC and anionic surfactant ADS in aqueous solutions have been measured by SANS, PGSE-NMR (self-diffusion coefficients), and DLS. Three methods yielded slightly different micelle diameters: for OlAC micelles, 6I $\AA$ (SANS), $96 \AA$ (PGSENMR); for ADS micelles, $41 \AA$ (SANS), $31 \AA$ (PGSENMR), and $25 \AA$ (DLS).

The shape of micelles is spherical, which is confirmed by the SANS data satisfying the Porod's law, $I_{s}(q) \propto q^{-4}$. The intermicellar distance with increasing surfactant concentration becomes smaller due to stronger repulsive interactions between micelles at high concentrations. Although the intermicellar distance becomes almost constant above 200 $\mathrm{mM}$, the structural transition of spherical micelles to cylindrical ones was not observed up to $300 \mathrm{mM}$ surfactant concentrations. DLS results also support for no structural transition of micelles over the concentrations examined.

Acknowledgements. This study was supported by Korea Institute of Science \& lechnology Evaluation and Planning 
(KISTEP) and Ministry of Science \& Teclunology (MOST). Korean government through its National Nuclear Technology Progrant.

H. U. Kim was supported by Korea Research Foundation (Program No: EN0037) for this work. Advices by Dr. B.-S. Seong and Y.-S. Han of Hanaro, Korea Atomic Energy Research Institute for SANS. Dr. C.-J. Cheong and E.-H. Kim of Korea Basic Science Institute for PGSE-NMR. and Bomoon Trading Co. for DLS are greatly appreciated.

\section{References}

1. Courchene. W. L. J. Phws Chent 1964,68,1870

2. Iwadare. Y. Suzawa. T. Nippon Kagaki Zasshi 1969.90, 1106.

3. Benedough, D.: Chen. S.-H.: Koeler, W. C. J. Phys. Chem. 1983. 87.2621

4. Chevalier. Y: Chachaty. C. Colloid Polm $S_{c i}$ 1984. 262. 489.

5. Misselyn-Bauduin. A.-M.: Thibaut. A.: Grandjean. J.: Broze. G.: Jerome R. J. Colloid Interface Sci 2001. 238, 1.

6. Walther. K. L.: Gradzielski. M.: Hotfmann. H.; Wakaum. A. $J$. Colloid Interface Sici. 1992. 153. 272.

7. Kang. K.-H.: Kim. H.-U.: Lim. K.-H. Colloid Surf .t 2001. 189. 113 .

8. Armarego. W. L. F.: Perrin. D. D. In Purification of Laboratory Chemicals, 4th ed.: Butterworth-Heinemann: Oxford, 1996

9. Korea Atomic Energy Research Institute, In Compung Guide for Hanaro Snall-Angle Scattering Data Treatment. 2002.

10. Guinier. A.: Foumet. G. In Smah-Angle Scattering of I-Rays: John Wiley \& Sons: New York. 1955.

11. Feigin. L. A.: Svergun. D. I. In Struchre Anatysis by Small-Angle A-Ray and Neutron Scattering: Taylor, G. W., Ed.; Plenum Press: New York and London, 1987: pp 68-69.

12. Porod, G. In Snall-Angle 1-Ran Scattering: Glatter O.: Krathy. O. Ed: Academic Press: New York. 1982: p 17.
13. Pedersen. T. S.: Egelhaaf. S. U.: Schurtenberger. P. I. Plys. Chem. 1995. 99. 1299.

14. Lin. M. Y.: Hanley, H. J. M.; Sinha, S. K.: Straty, G. C.: Peiffer, D. G. Kim. M. W. Phwsica B 1995. 213-211,613

15. Holtzer. A. J. Pohn. Sci. 1995, 17.432.

16. Denkinger. P.: Burchard. W. J. Polym. Sci. B. Polmm. Phws. 1991. 29.589 .

17. Kato. T. J. Plns. Chem. 1985. 89.5750.

18. Braun. S.; Kalinowski, H.-O.: Berger, S. In 100 and More Basic $M R$ Experintents; A Practical Conse; VCH: 1999: Ch.11, pp 349-351.

19. Lindman. B.: Olsson. U.: Soderman. O. In Handbook of Hicroemulsion Science and Techologv: 1998: Ch. 10. pp 309-356.

20. Walderhaug. H.: Nystrom. B. Colloid Suff A 1999. 149.379.

21. Annunziata, O.: Costantino. L.: D'Errico, G.: Paduano, L.: Vitagliano, V. J. Colloidinterface Sici. 1999. 216, 16

22. Caria A.: Regev. O.: Khan, A. J. Colloid Interface Sci, 1998, 200. 19

23. Bruker Co.. Almanac. 2001: $\mathrm{p} 72$.

24. Weingartter. H. Z. Plys. Chem. Aeue Folge। 1982. 132.129.

25. Holz. M. Weingartner, H. J. Magn. Reson 1991, 92.115

26. Israelachvili. J. N. In Internolecular and Sinface Forces. $2^{\text {nd }}$ Ed. Academic Press: London. 1991.

27. Tanford. C. J. Phus. Chem. 1974. 78.2469

28. Kim. H.-U. Ph.D Dissertation: Chung-Ang University: Korea. 2002.

29. Jonsson1. B.: Lindmant1. B.: Holmberg. K.: Kronberg. B. In Surfactants and Polnters in Aqueous Solutions: John Wiley and Sons: New York. 1997; pp 84-100.

30. Cabane. B.: Duplessix. R. Zemb, T. J. Phos 1985, +6. 2161.

31. Fprland. G. M.: Sameth. J.: Gjerde. M. I.: Hfiland. H.: Jensen. A. F.: Mortensen. K. J. Colloid hiterface Sci. 1998. 203.328.

32. Grabner. D.: Matsuo. T.: Hoinkis. E.: Thunig. C.: Hoffmann1. H. $J$ Colloid Interface Sci, 2001, 236. 1.

33. Kim. H. U. Ph. D. Dissentation: Chung-Ang University: Seoul. Korea. 2002: pl66.

34. Reiss-Husson. F. Luzzati. V. I Plyss. Chem 1964.86,3504. 